\title{
Assessment of genetic factor and depression interactions for asthma symptom severity in cohorts of childhood and elderly asthmatics
}

Heung-Woo Park $\mathbb{B}^{1,2}$, Woo-Jung Song², Sang-Heon Cho², Michael J. McGeachie', Fernando Martinez ${ }^{3}$, Dave Mauger ${ }^{4}$, Bruce G. Bender ${ }^{5}$ and Kelan G. Tantisira,

\begin{abstract}
It is well known that depression is associated with asthma symptoms. We assessed the combined effects of genetic factors and depression on asthma symptom severity using Bayesian network (BN) analysis. The common 100 topranked single-nucleotide polymorphisms (SNPs) were obtained from two genome-wide association studies of symptom severity in two childhood asthmatics trials (CAMP (Childhood Asthma Management Program) and CARE (Childhood Asthma Research and Education)). Using SNPs plus five discretized variables (depression, anxiety, age, sex, and race), we performed BN analysis in 529 CAMP subjects. We identified two nodes (depression and rs4672619 mapping to ERBB4 (Erb-B2 receptor tyrosine kinase 4)) that were within the Markov neighborhood of the symptom node in the network and then evaluated the interactive effects of depressive status and rs4672619 genotypes on asthma symptom severity. In childhood asthmatics with homozygous reference alleles, severe depression was related to less severe symptoms. However, in childhood asthmatics with heterozygous alleles and homozygous variant alleles, depression and symptom severity showed a positive correlation (interaction permutation $P$ value $=0.019$ ). We then tried to evaluate whether the interactive effects that we found were sustained in another independent cohort of elderly asthmatics. Contrary to the findings from childhood asthmatics, elderly asthmatics with homozygous reference alleles showed a positive correlation between depression and symptom severity, and elderly asthmatics with heterozygous alleles and homozygous variant alleles showed a negative correlation (interaction permutation $P$ value $=0.003$ ). In conclusion, we have identified a novel SNP, rs4672619, that shows interactive effects with depression on asthma symptom severity in childhood and elderly asthmatics in opposite directions.
\end{abstract}

\section{Introduction}

A depressive disorder may influence the symptoms present in the course of a chronic illness. For example, the presence of depression was shown to be a stronger predictor of reporting diabetes symptoms than hemoglobin

Correspondence: Heung-Woo Park (guinea71@snu.ac.kr)

'Department of Medicine, Channing Division of Network of Medicine, Brigham and Womens Hospital, Harvard Medical School, Boston, MA, USA

${ }^{2}$ Department of Internal Medicine, Seoul National University College of

Medicine, Seoul, Republic of Korea

Full list of author information is available at the end of the article.
$\mathrm{A}_{1 \mathrm{C}}$ level and was more highly associated with chest pain than objective measures of coronary artery disease severity ${ }^{1,2}$. Asthma is not an exception. Epidemiologic associations between depression and asthma symptoms have been recognized anecdotally for many years ${ }^{3-5}$. Dysregulation in key biologic systems, such as inflammation $^{6}$, neuroendocrine dysregulation ${ }^{7}$, autonomic imbalance causing cholinergic activation ${ }^{8}$, and genetics dysregulation ${ }^{9}$, have been suggested as explanations of this co-occurrence.

\section{○) The Author(s) 2018}

(c) (i) Open Access This article is licensed under a Creative Commons Attribution 4.0 International License, which permits use, sharing, adaptation, distribution and reproduction cc) in any medium or format, as long as you give appropriate credit to the original author(s) and the source, provide a link to the Creative Commons license, and indicate if changes were made. The images or other third party material in this article are included in the article's Creative Commons license, unless indicated otherwise in a credit line to the material. If material is not included in the article's Creative Commons license and your intended use is not permitted by statutory regulation or exceeds the permitted use, you will need to obtain permission directly from the copyright holder. To view a copy of this license, visit http://creativecommons.org/licenses/by/4.0/. 
Table 1 Characteristics of childhood and elderly asthmatics

\begin{tabular}{lll}
\hline & Childhood asthmatics, $\mathbf{N}=\mathbf{2 6}$ & Elderly asthmatics, $\mathbf{N = \mathbf { 9 6 }}$ \\
\hline Age (years), median (IQR) & $8.8(7.2-10.6)$ & $72.5(70-77)$ \\
Male gender, number (\%) & $332(62.7)$ & $38(39.6)$ \\
Symptom score, median (IQR) & $0.57(0.14-1.00)^{\mathrm{a}}$ & $8(6-10)^{\mathrm{b}}$ \\
Race, number (\%) & & $0(0) / 0(0) / 0(0) / 96(100)$ \\
Non-Hispanic white/African American/Hispanic/Asian & $389(73.6) / 58(10.9) / 40(7.6) / 42(7.9)$ & $27(24-29)^{\mathrm{c}}$ \\
Cognitive function (score), median (IQR) & $\mathrm{NA}$ & $\mathrm{NA}$ \\
Anxiety (score), median (IQR) & $10(6-15)$ & $19.5(16-22)$ \\
Depression (score), median (IQR) & $5(2-10)$ & \\
\hline
\end{tabular}

$I Q R$ interquartile range, $N A$ not applicable

${ }^{\text {a Range } 0-3}$

${ }^{\mathrm{b}}$ Range 0-25

'Range, $0-30$ (any score $\geq 24$ points indicates normal cognition)

A Bayesian network (BN) is a multivariate model of dependency among several variables, and the strength of the relationship between variables is represented by conditional probability distributions associated with each node ${ }^{10,11}$. BNs have been applied in a variety of settings for the purposes of probabilistic prediction, including predictions of asthma exacerbations and control ${ }^{12,13}$ and responses to short-acting bronchodilator and inhaled corticosteroids $^{14,15}$. Given that depression is associated with asthma symptoms, we hypothesized that combining information from multiple SNPs, demographic factors, depression, and anxiety can yield models predictive of symptoms. Using BNs, we developed a predictive model and identified one candidate single-nucleotide polymorphism (SNP), rs4672619, that showed possible combined effects with depression upon asthma symptoms. Rs4672619 is located on the intron of the Erb-B2 receptor tyrosine kinase 4 (ERBB4) gene and showed interactive effects with depression on asthma symptom severity in children from the Childhood Asthma Management Program (CAMP) trial ${ }^{16}$. We confirmed that interactions between depression and rs4672619 in predicting asthma symptom severity were also noted in an independent cohort of elderly asthmatics, although the interactions were in the opposite direction.

\section{Materials and methods}

Each study was approved by the Institutional Review Board of the corresponding institution and informed consent was obtained from all study participants. Detailed methods are described in the supplementary material.

\section{Identifying SNPs associated with asthma symptoms}

To obtain reliable SNPs associated with asthma symptoms, we used the results of two genome-wide association study (GWAS) performed in the non-Hispanic white children with asthma enrolled in the CAMP trial $(n=438)$ and in the Childhood Asthma Research and Education (CARE) trials $(n=457)$. At baseline, all participants were asked to rate and score their asthma symptoms during the past $24 \mathrm{~h}$ on a diary card. Similar questions were used in both trials, and the symptom scores ranged from 0 (absent) to 3 (severe) in all trials ${ }^{16-}$ 18. Detailed methods of genome-wide SNP genotyping have been described elsewhere for CAMP ${ }^{19,20}$ and $\mathrm{CARE}^{21}$. A common set of SNP genotypes was obtained by imputation in each cohort using $\mathrm{MaCH}$ (version 1.0) ${ }^{22}$ and the 1000 Genomes Project EUR reference-phased haplotypes based on Phase 1 low coverage data (20101123 release). SNPs with minor allele frequency $<1 \%$, a Hardy-Weinberg equilibrium $P<0.001$, and/or an imputation quality score $<0.3$ were excluded, resulting in a set of $\sim 37$ million variants per cohort. These data and quality control have been described previously ${ }^{23}$. The association of SNPs with asthma symptom scores at baseline was measured with a linear regression model as implemented in $\mathrm{PLINK}^{24}$ using additive genetic models. The regression models were adjusted for age and sex. From results of two GWAS, common SNPs with $P$ values $<0.05$ showing same directionality were obtained. As information on depression and anxiety was available only in the CAMP trial, $\mathrm{BN}$ analysis was performed in the CAMP cohort. Among common SNPs, the 100 topranked SNPs based on $P$ values of CAMP were forwarded to $\mathrm{BN}$ analysis.

\section{BN analysis}

We view the $\mathrm{BN}$ as primarily a hypothesis-generating step in the analysis. In the CAMP trial, measures of depression and anxiety status were collected at baseline and annually thereafter using the Children's Depression Inventory $^{25}$ and the Revised Children's Manifest 


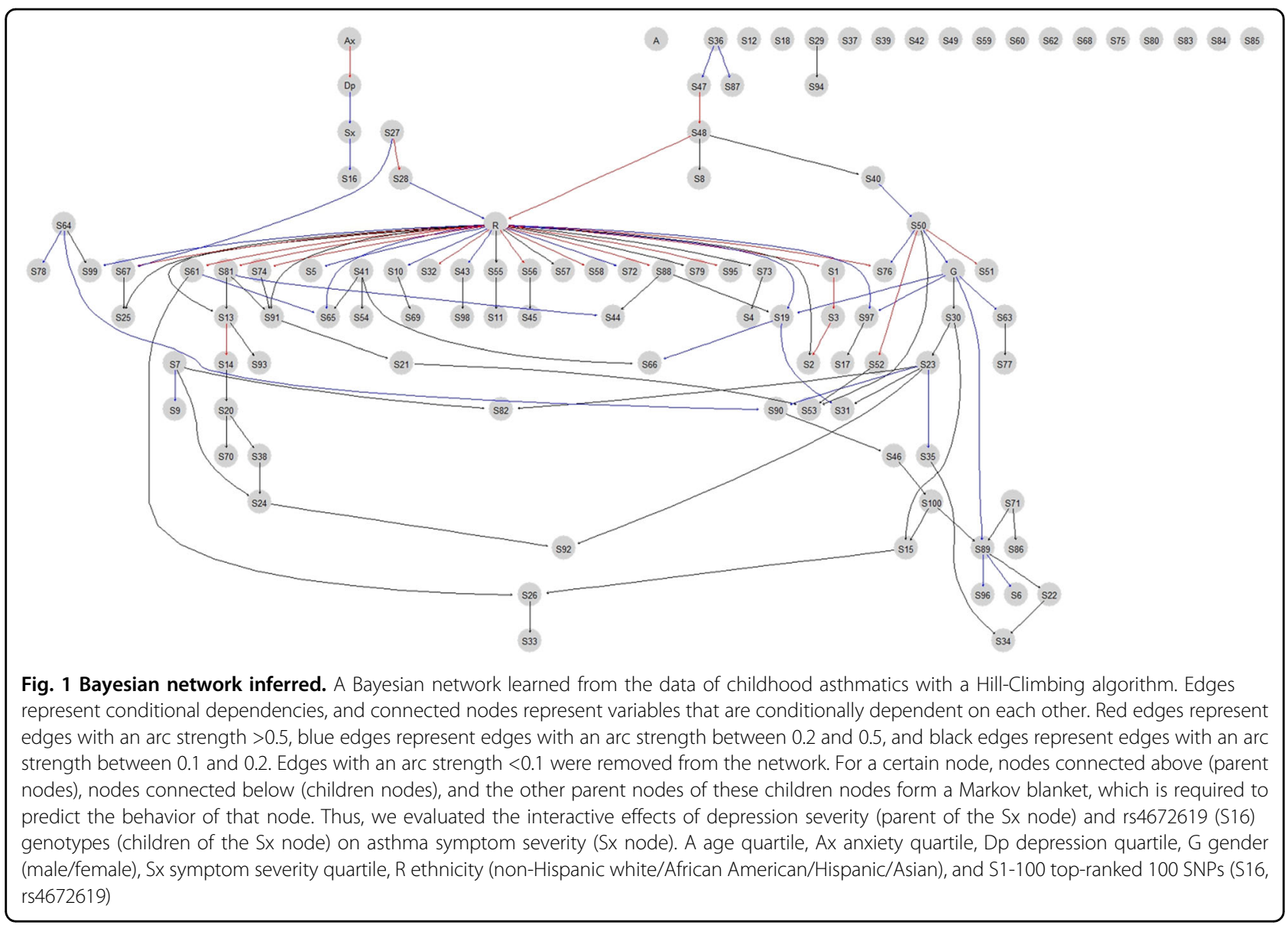

Anxiety ${ }^{26}$. Higher scores on each of these measures reflect increased problems. For better modeling, we drew 91 subjects with other ethnicities from the CAMP trial in addition to the 438 non-Hispanic white subjects, and resultantly, a total of 529 subjects who had a complete phenotype data set (symptom, depression, and anxiety scores) and genotype data were used for $\mathrm{BN}$ analysis. The baseline characteristics are presented in Table 1. We used the open-source software package bnlearn ${ }^{27}$ in the statistical and graphical environment $\mathrm{R}$ (http://www.rproject.org) for $\mathrm{BN}$ analysis. In $\mathrm{BN}$ analysis, we included 105 variables: 100 SNPs (homozygous minor alleles and heterozygous and homozygous major alleles); depression score (quartile 1-4); anxiety score (quartile 1-4); age (quartile 1-4); sex (male and female); and race (nonHispanic white, African American, any Hispanic, and Asian). Prior distributions used were bnlearn defaults, and we learned a BN structure using bnlearn's hill-climbing algorithm (a greedy optimizer for the Bayesian posterior probability of the data). Measuring the degree of confidence in a particular graphical feature is a key problem in inferring the network structure ${ }^{28}$. We quantified such a degree of confidence by generating 1000 network structures based on nonparametric bootstrap to the data and by estimating the relative frequency of the feature of interest ${ }^{28}$. Then, we removed undirected arcs and arcs with strength $<0.1$ from the $\mathrm{BN}$ inferred. A previous report showed that removing the arcs with strength $<0.1$ had a minimal effect on its classification accuracy ${ }^{29}$. According to the Markov blanket property of the BN, only the parents (nodes connected above), children (nodes connected below), and the other parents of those children are required to predict the behavior of a node ${ }^{30}$. We therefore selected the two nodes (depression and rs4672619) that were within the Markov blanket of the symptom node in $\mathrm{BN}$ and then performed interaction analysis. To calculate an adjusted $P$ value, permutation tests for a linear model as implemented in the "lmPerm" $R$ package $^{31}$ was done.

\section{Evaluating the interactive effects in another cohort}

We evaluated whether the interactive effects of depression status and rs4672619 genotypes on asthma symptom severity observed in the cohort of childhood asthmatics were sustained in another independent cohort. As no childhood cohort with both depression and genotype data was readily available, our evaluation was done in elderly asthmatics aged 65 years or older from a 
prospective, observational, and multi-centered cohort in Korea with the purpose of studying the natural history of asthma among elderly people ${ }^{32}$. The baseline characteristics are presented in Table 1 . Symptom scores were measured using five questionnaires (range, 0-25; a higher score represents a more severe symptom), and depression status was assessed by the Korean version of the Geriatric Depression Scale Short Form (range, 0-30; a higher score represented more severe depression). Cognitive function was assessed using the Korean version of the Mini-Mental State Examination (range, $0-30$; any score $\geq 27$ points indicates a normal cognition). Details for symptom questionnaires are described in the online supplement. rs4672619 was genotyped, and the interactive effects between genotype and depression status on asthma symptom severity were evaluated.

\section{Results}

The lowest $P$ value for GWAS analysis was $1.66 \times 10^{-6}$ (rs2141189) in the CAMP trial and $1.52 \times 10^{-6}$ (rs1429146) in the CARE trial. There were 413 common SNPs with $P$ values $<0.05$ with the same directionality. Of these SNPs, the 100 top-ranked SNPs based on the CAMP $P$ values (Supplementary Table 1 ) were selected and forwarded for BN analysis. Figure 1 shows the BN learned from the data of childhood asthmatics. We found two nodes directly attached to the asthma symptom ( $\mathrm{Sx}$ node in Fig. 1): depression (Dp node in Fig. 1) and rs4672619 (S16 node in Fig. 1). The arc strengths between the Sx and Dp nodes and between the Sx and S16 nodes were 0.329 and 0.207 , respectively. We then evaluated the interactive effects of depression severity and rs4672619 genotypes on asthma symptom severity in all CAMP subjects. As shown in Fig. 2a, in childhood asthmatics with homozygous reference alleles (frequency $=86.8 \%$ ), severe depression was related to less severe asthma symptoms. However, in childhood asthmatics with heterozygous or homozygous variant alleles, depression and asthma symptom severity showed a positive correlation. The $P$ values of the main effects were 0.009 for rs 4672619 and 0.322 for depression, and the $P$ value of the interaction was 0.014 (permutation $P$ value $=0.019$ ). rs4672619 was genotyped in 96 Korean elderly asthmatics, and we also found that the directions of correlations between depression status and asthma symptom severity differed according to rs4672619 genotypes (Fig. 2b). However, the direction of correlation was opposite to that of childhood asthmatics (Fig. 2). That is, elderly asthmatics with homozygous reference alleles (frequency $=92.4 \%$ ) showed a positive correlation between depression and symptom severity. The $P$ values of the main effects were 0.177 for rs4672619 and 0.125 for depression, and the $P$ value of the interaction was 0.00014 (permutation $P$ value $=0.003)$.

\section{Discussion}

Precise symptom perception would be an important component of asthma management, allowing asthmatics to use rescue medicine or seek medical help in a timely manner ${ }^{33}$. However, there may be a discrepancy between asthma symptom perception and lung function ${ }^{34}$. Therefore, asthma symptoms are a unique domain that should be evaluated for the proper management of asthma $^{35}$. $\beta 2$ Adrenergic receptor gene methylation was reported to be associated with decreased asthma severity in inner-city schoolchildren ${ }^{36}$, and genetic components are known to be involved in asthma symptom responses to medications ${ }^{37,38}$. In addition, a recent report showed that psychosocial factors, such as harsh parent-child conflict $^{39}$ or targeted rejection ${ }^{40}$, caused severe symptoms in children with asthma by decreasing anti-inflammatory gene expression. These findings motivated us to assess the interactive effects of multiple SNPs mapped to multiple genes and depression severity on asthma symptoms. Using BNs to guide our investigation, we found that rs4672619 was directly attached to the symptom node. Further analysis has shown that rs4672619 genotypes showed significant interactive effects with depression severity on symptom severity in both childhood and elderly asthmatics.

rs4672619 is on an intron of the ERBB4 gene. ERBB4, along with its ligand neuregulin-1, is known to contribute to the pathophysiology of schizophrenia and bipolar disorders $^{41,42}$. A recent Bayesian modeling approach identified several variants in ERBB4 with strong evidence for associations with childhood asthma ${ }^{43}$. Recent increases in the understanding of dyspnea, one of the important asthma symptoms, suggest that there may be interactions between biopsychological factors and dyspnea perception $^{44}$. ERBB4 regulates a thalamic reticular nucleus to cortical inputs at levels that can support sensory selection while allowing behavioral flexibility ${ }^{45}$. In addition, crosstalk between the ErbB network and steroid hormone signaling pathway has been well known ${ }^{46}$ and severe, steroid-dependent asthmatics had depression more often than non-steroid-dependent asthmatics ${ }^{47}$. Taken together, rs4672619 is possibly involved in interactions between depression and asthma symptom severity by modulating symptom perception and steroid action.

One of the interesting findings of this study was that the direction of interactive effects of rs4672619 genotypes with depression severity on asthma symptoms was opposite between our childhood and elderly cohorts. The childhood asthmatics carrying two reference alleles showed significantly negative correlations between depression and symptoms scores, whereas elderly asthmatics with these alleles showed significantly positive correlations. Cognitive dysfunction, which is frequently encountered in elderly asthmatics ${ }^{48}$, might affect asthma 
A

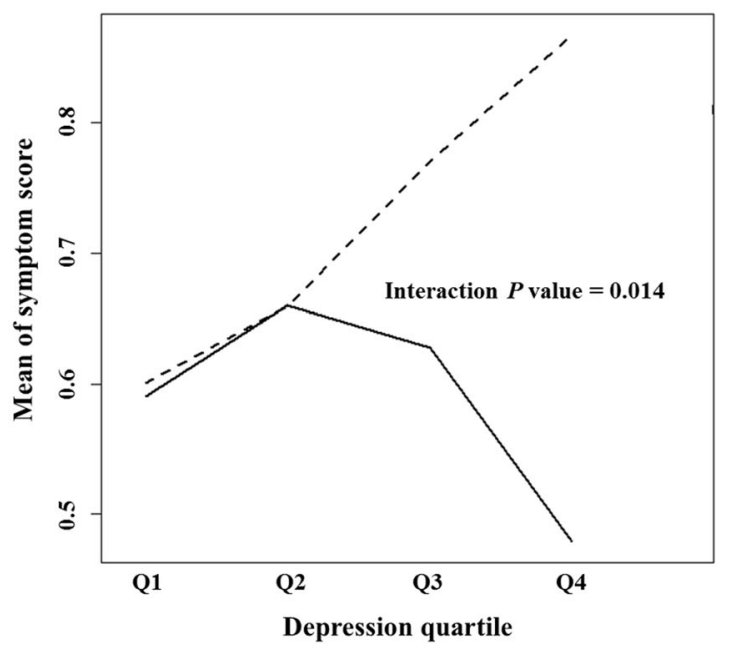

B

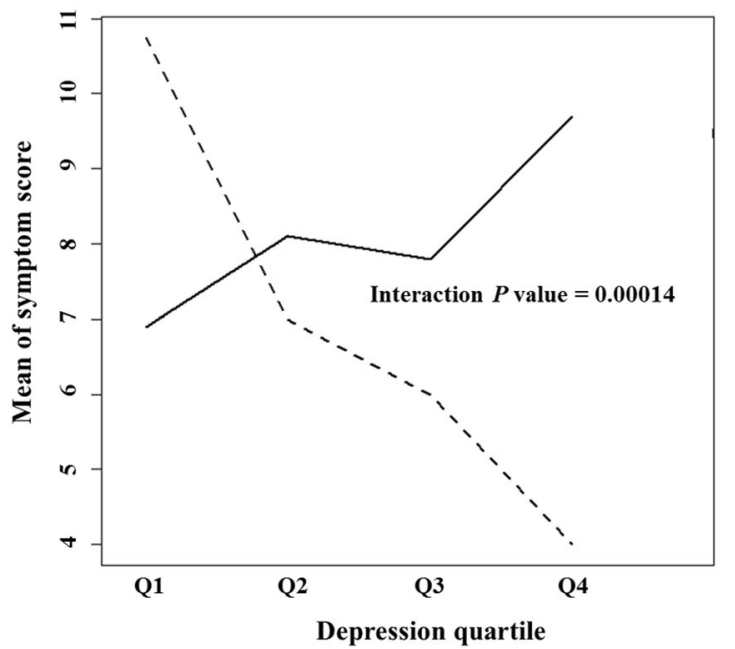

Fig. 2 Interactive effects of rs4672619 genotypes and depression severity on asthma symptom severity in cohorts of childhood and elderly asthmatics. a Childhood asthmatics. b Elderly asthmatics. Solid lines represent subjects with reference homozygous alleles of rs 4672619 , and dotted lines represent subjects with heterozygous or homozygous variant alleles of rs4672619. Q1 represents mild depression, and Q4 represents severe depression. The severity of asthma symptoms increases as symptom score increases

symptom presentation. However, we found that the MiniMental State Examination scores of all elderly asthmatics were greater than 24 (Table 1), which suggested that they were cognitively competent ${ }^{49}$. Many investigators have agreed that elderly asthma differs considerably from nonelderly asthma and that aging lungs ${ }^{50}$, physiologic changes $^{51}$, and immunosenescence ${ }^{52}$ are possible contributing factors. Likewise, the different interactive effects of rs4672619 genotypes and depression severity on asthma symptom severity between childhood and elderly asthmatics might come from the factors mentioned above. It is also possible that asthma symptom perception may be influenced by age, and a specific genetic mechanism regulating childhood asthma symptom severity may not carry over to old age. To date, no study has evaluated differences in the perception of asthma symptoms in childhood and elderly asthmatics. However, for childhood asthmatics, it was reported that adolescents (13-18 years) were more accurate in perceiving symptoms than schoolage children $(6-12 \text { years })^{31}$ and that the accuracy for perceiving symptoms increased with age in children aged 7 to 17 years $^{53}$. In our previous study, we found that genetic mechanisms underlying the symptomatic response to inhaled corticosteroids might be different between childhood and adult asthmatics ${ }^{36}$. Interestingly, recent reports showed that some genetic variations in $E R B B 4$ showed age-by-genotype interactions on cortical brain morphology in a cohort of 3-20 year olds ${ }^{54}$, and age-dependent differences in ERBB4 expression were found in the brains of autistic patients ${ }^{55}$. These findings suggest that the genetic effects of ERBB4 may be different between childhood and elderly asthmatics, although we do not know whether the same differences exist in the lung.

Another plausible explanation is that the different interactive effects are due to ethnicity differences. Childhood asthmatics in this study were predominantly nonHispanic white patients, and whereas elderly asthmatics were only Asian patients. Allele reversal at shared risk loci, the so-called flip-flop phenomenon, can be attributed to differences in the underlying genomic architectures at these loci according to ethnicity differences ${ }^{56}$. For example, a previous report showed that variants of ENND1B were associated with asthma in children, but the association in the African Americans was linked to the opposite allele of that associated with asthma in subjects of European ancestry ${ }^{57}$. Very little is known about ethnicity-gene interactions for ERBB4. Only one small-scale study conducted in non-Hispanic white and African-American schizophrenia patients reported that disease-associated SNPs on ERBB4 showed no ethnicity-genotype effects on ERBB4 splice-variant expression levels in the brain ${ }^{58}$.

There are a few potential limitations in generalizing our findings. Inevitably, the main effect and interaction effect are often correlated. It is possible that the significant effect of rs4672619 genotypes might have affected the interactive effect of depression severity and rs4672619 genotypes on asthma symptom severity in childhood asthmatics. Next, we could not replicate our results in another pertinent cohort of childhood asthmatics. The opposite directions of the correlations in childhood and 
elderly asthmatics was interesting. However, we cannot completely exclude the possibility that our results may be due to chance alone (false-positive associations). In addition, further mechanistic studies are needed to confirm the age-specific or ethnicity-specific effects of rs4672619 on the ERBB4 gene. An incorporation of prior biological knowledge would help us overcome the reconstruction accuracy due to the complex nature of the network and the noise inherent in the data.

In conclusion, we have identified rs4672619, which showed significant interactive effects with depression severity on asthma symptom severity in both childhood and elderly asthmatics in opposite directions. This study is the first to show significant interactions between genetic factors and depression severity for asthma symptom severity. Our findings suggest that different strategies to decrease asthma symptom severity may be needed in these distinct age subsets of asthmatics.

\section{Acknowledgements}

This work was supported by the National Institutes of Health, US (R01 NR013391, R01 HL127332, and U01 HL065899) to K.G.T., by the Ministry of Health and Welfare, Republic of Korea (2008-E33028-00, 2009-E33022-00, and 2011-E33005-00) to H.-W.P., and by the Parker B. Francis Foundation to M.J.M.

\section{Author details}

'Department of Medicine, Channing Division of Network of Medicine, Brigham and Womens Hospital, Harvard Medical School, Boston, MA, USA. ${ }^{2}$ Department of Internal Medicine, Seoul National University College of Medicine, Seoul, Republic of Korea. ${ }^{3}$ Arizona Respiratory Center, University of Arizona, Tucson, AZ, USA. ${ }^{4}$ Department of Public Health Sciences, Pennsylvania State University College of Medicine, Hershey, PA, USA. ${ }^{5}$ National Jewish Health, Denver, CO, USA. ${ }^{6}$ Department of Medicine, Division of Pulmonary and Critical Care Medicine, Brigham and Women's Hospital, Boston, MA, USA

\section{Authors' contributions}

H.-W.P. and K.G.T.: Conception and design of the study, data generation, analysis, interpretation, and preparation of the manuscript. W.-J.S. and S.-H.C. Data generation and critical revision of the manuscript. M.J.M.: Data generation, analysis, interpretation, and preparation of the manuscript. F.M. D.M., and B.G.B.: Data generation, analysis, interpretation, and critical revision of the manuscript.

\section{Conflict of interest}

The authors declare that they have no conflict of interest.

\section{Publisher's note}

Springer Nature remains neutral with regard to jurisdictional claims in published maps and institutional affiliations.

Supplementary information accompanies this paper at https://doi.org/ 10.1038/s12276-018-0110-5.

Received: 31 May 2017 Revised: 26 March 2018 Accepted: 30 March 2018 Published online: 04 July 2018

\footnotetext{
References

1. Ludman, E. J. et al. Depression and diabetes symptom burden. Gen. Hosp. Psychiatry 26, 430-436 (2004).

2. Ruo, B. et al. Depressive symptoms and health-related quality of life: the Heart and Soul Study. JAMA 290, 215-221 (2003).
}

3. Janson, C., Bjornsson, E., Hetta, J. \& Boman, G. Anxiety and depression in relation to respiratory symptoms and asthma. Am. J. Respir. Crit. Care Med. 149, 930-934 (1994).

4. Rimington, L. D., Davies, D. H., Lowe, D. \& Pearson, M. G. Relationship between anxiety, depression, and morbidity in adult asthma patients. Thorax 56, 266-271 (2001)

5. Goldney, R. D., Ruffin, R., Fisher, L. J. \& Wilson, D. H. Asthma symptoms associated with depression and lower quality of life: a population survey. Med. J. Aust. 178, 437-441 (2003).

6. Shanahan, L., Copeland, W. E., Worthman, C. M., Angold, A. \& Costello, E. J. Children with both asthma and depression are at risk for heightened inflammation. J. Pediatr. 163, 1443-1447 (2013).

7. Van Lieshout, R. J., Bienenstock, J. \& MacQueen, G. M. A review of candidate pathways underlying the association between asthma and major depressive disorder. Psychosom. Med. 71, 187-195 (2009).

8. Miller, B. D. \& Wood, B. L. Influence of specific emotional states on autonomic reactivity and pulmonary function in asthmatic children. J. Am. Acad. Child Adolesc. Psychiatry 36, 669-677 (1997).

9. Wamboldt, M. Z., Hewitt, J. K. \& Schmitz, S. Familial association between allergic disorders and depression in adult Finnish twins. Am. J. Med. Genet. 96 146-153 (2000).

10. Shoemaker, J. S., Painter, I. S. \& Weir, B. S. Bayesian statistics in genetics: a guide for the uninitiated. Trends Genet 15, 354-358 (1999).

11. Beaumont, M. A. \& Rannala, B. The Bayesian revolution in genetics. Nat. Rev. Genet. 5, 251-261 (2004).

12. Farion, K. J., Wilk, S., Michalowski, W., O'Sullivan, D. \& Sayyad-Shirabad, J. Comparing predictions made by a prediction model, clinical score, and physicians: pediatric asthma exacerbations in the emergency department. Appl. Clin. Inform. 4, 376-391 (2013).

13. McGeachie, M. J. et al. The metabolomics of asthma control: a promising link between genetics and disease. Immun. Inflamm. Dis. 3, 224-238 (2015).

14. Himes, B. E. et al. Predicting response to short-acting bronchodilator medication using Bayesian networks. Pharmacogenomics 10, 1393-1412 (2009).

15. McGeachie, M. J. et al. Predicting inhaled corticosteroid response in asthma with two associated SNPs. Pharm. J. 13, 306-311 (2013).

16. Childhood Asthma Management Program Research Group. The Childhood Asthma Management Program (CAMP): design, rationale, and methods. Control Clin. Trials 20, 91-120 (1999).

17. Szefler, S. J. et al. Characterization of within-subject responses to fluticasone and montelukast in childhood asthma. J. Allergy Clin. Immunol. 115, 233-242 (2005).

18. Sorkness, C. A. et al. Long-term comparison of 3 controller regimens for mildmoderate persistent childhood asthma: the Pediatric Asthma Controller trial. J. Allergy Clin. Immunol. 119, 64-72 (2007).

19. Murphy, A. et al. Mapping of numerous disease-associated expression polymorphisms in primary peripheral blood CD4+ lymphocytes. Hum. Mol. Genet 19, 4745-4757 (2010).

20. Himes, B. E. et al. Genome-wide association analysis identifies PDE4D as an asthma-susceptibility gene. Am. J. Hum. Genet. 84, 581-593 (2009).

21. Torgerson, D. G. et al. Meta-analysis of genome-wide association studies of asthma in ethnically diverse North American populations. Nat. Genet. 43, 887-892 (2011).

22. Li, Y., Willer, C. J., Ding, J., Scheet, P. \& Abecasis, G. R. MaCH: using sequence and genotype data to estimate haplotypes and unobserved genotypes. Genet. Epidemiol. 34, 816-834 (2010).

23. Croteau-Chonka, D. C. et al. Expression quantitative trait loci information improves predictive modeling of disease relevance of non-coding genetic variation. PLOS ONE 10, e0140758 (2015).

24. Purcell, S. et al. PLINK: a tool set for whole-genome association and population-based linkage analyses. Am. J. Hum. Genet. 81, 559-575 (2007).

25. Kovacs, M. Rating scales to assess depression in school aged children. Acta Paedopsychiatr. 46, 305-318 (1981).

26. Reynolds, C. \& Richmond, B. Revised Children's Manifest Anxiety Scale (RCMAS) (Western Psychological Services, Los Angeles, CA, 1985).

27. Scutari, M. Learning Bayesian networks with the bnlearn R package. J. Stat. Softw. 35, 1-22 (2010)

28. Friedman, N., Goldszmidt, M., Wyner, A. Data analysis with Bayesian networks: a Bootstrap approach. In Proc 15th Conference on Uncertainty in Artificial Intelligence 206-215 (Morgan Kaufmann: San Francisco, CA, 1999).

29. Ratnapinda, P. \& Druzdzel, M. J. An empirical evaluation of costs and benefits of simplifying Bayesian networks by removing weak arcs. In Proc 27th 
International Florida Artificial Intelligence Research Society Conference 508-511 (AAAl Press, Palo Alto, CA, 2014).

30. Ghosh, J. K., Delampady, M. \& Samanta, T. An Introduction to Bayesian Analysis Theory and Methods. Springer Texts in Statistics (Springer: New York, NY, 2006).

31. Wheeler B. ImPerm:Permutational tests for linear models. 2010. Available at: http://CRAN.R-project.org/package $=$ ImPerm.

32. Park, H. W. et al. Prediction of asthma exacerbations in elderly adults: results of a 1-year prospective study. J. Am. Geriatr. Soc. 61, 1631-1632 (2013).

33. Yoos, H. L. \& McMullen, A. Symptom perception and evaluation in childhood asthma. Nurs. Res. 48, 2-8 (1999).

34. Park, H. W. et al. Xenon ventilation computed tomography and the management of asthma in the elderly. Respirology 19, 389-395 (2014).

35. The National Asthma Education and Prevention Program (NAEPP). National Asthma Education and Prevention Program Expert Panel Report 3: Guidelines for the Diagnosis and Management of Asthma 2007. http://www.nhlbi.nih. gov/guidelines/asthma/asthgdln.pdf. Accessed 8 Dec 2016.

36. Gaffin, J. M. et al. $\beta-2$ adrenergic receptor gene methylation is associated with decreased asthma severity in inner-city schoolchildren: asthma and rhinitis. Clin. Exp. Allergy 44, 681-689 (2014).

37. Park, H. W. et al. Genetic predictors associated with improvement of asthma symptoms in response to inhaled corticosteroids. J. Allergy Clin. Immunol. 133, 664-669 (2014).

38. Mougey, E. B. et al. Pharmacogenetics of asthma controller treatment. Pharm. J. 13, 242-250 (2013)

39. Ehrlich, K. B., Miller, G. E. \& Chen, E. Harsh parent-child conflict is associated with decreased anti-inflammatory gene expression and increased symptom severity in children with asthma. Dev. Psychopathol. 27, 1547-1554 (2015).

40. Murphy, M. L., Slavich, G. M., Chen, E. \& Miller, G. E. Targeted rejection predicts decreased anti-inflammatory gene expression and increased symptom severity in youth with asthma. Psychol. Sci. 26, 111-121 (2015).

41. Maier, W., Zobel, A. \& Wagner, M. Schizophrenia and bipolar disorder: differences and overlaps. Curr. Opin. Psychiatry 19, 165-170 (2006).

42. Wang, N. et al. Downregulation of neuregulin 1-ErbB4 signaling in parvalbumin interneurons in the rat brain may contribute to the antidepressant properties of ketamine. J. Mol. Neurosci. 54, 211-218 (2014).

43. Baurley, J. W. \& Conti, D. V. A scalable, knowledge-based analysis framework for genetic association studies. BMC Bioinform. 14, 312 (2013).
44. Hayen, A., Herigstad, M. \& Pattinson, K. T. Understanding dyspnea as a complex individual experience. Maturitas 76, 45-50 (2013).

45. Ahrens, S. et al. ErbB4 regulation of a thalamic reticular nucleus circuit for sensory selection. Nat. Neurosci. 18, 104-111 (2015).

46. Yarden, Y. \& Sliwkowski, M. X. Untangling the ErbB signalling network. Nat. Rev. Mol. Cell. Biol. 2, 127-137 (2001).

47. Amelink, M. et al. Anxiety, depression and personality traits in severe, prednisone-dependent asthma. Respir. Med. 108, 438-444 (2014).

48. Ng, T. P., Chiam, P. C. \& Kua, E. H. Mental disorders and asthma in the elderly: a population-based study. Int. J. Geriatr. Psychiatry 22, 668-674 (2007).

49. Tombaugh, T. N. \& Mclntyre, N. J. The Mini-Mental State Examination: a comprehensive review. J. Am. Geriatr. Soc. 40, 922-935 (1992).

50. Enright, P. L., Kronmal, R. A., Manolio, T. A., Schenker, M. B. \& Hyatt, R. E. Respiratory muscle strength in the elderly. Am. J. Respir. Crit. Care Med. 149 430-438 (1994).

51. Knudson, R. J., Lebowitz, M. D., Holberg, C. J. \& Burrows, B. Changes in the normal maximal expiratory flow volume curve with growth and aging. Am. Rev. Respir. Dis. 127, 725-734 (1983).

52. Agarwal, S. \& Busse, P. J. Innate and adaptive immunosenescence. Ann. Allergy Asthma Immunol. 104, 183-190 (2010).

53. Kopel, S. J. et al. Asthma symptom perception and obesity in children. Biol. Psychol. 84, 135-141 (2010).

54. Douet, V., Chang, L., Lee, K. \& Ernst, T. Pediatric Imaging, Neurocognition, and Genetics (PING) Consortium. ERBB4 polymorphism and family history of psychiatric disorders on age-related cortical changes in healthy children. Brain Imag. Behav. 9, 128-140 (2015).

55. Chow, M. L. et al. Age-dependent brain gene expression and copy number anomalies in autism suggest distinct pathological processes at young versus mature ages. PLoS Genet. 8, e1002592 (2012).

56. Lin, P. I., Vance, J. M., Pericak-Vance, M. A. \& Martin, E. R. No gene is an island: the flip-flop phenomenon. Am. J. Hum. Genet. 80, 531-538 (2007).

57. Sleiman, P. M. et al. Variants of DENND1B associated with asthma in children. N. Engl. J. Med. 362, 36-44 (2010).

58. Law, A. J., Kleinman, J. E., Weinberger, D. R. \& Weickert, C. S. Diseaseassociated intronic variants in the ErbB4 gene are related to altered ErbB4 splice-variant expression in the brain in schizophrenia. Hum. Mol. Genet. 16, 129-141 (2007). 